\title{
Hypertrophic Cardiomyopathy with a Twist
}

\author{
W. Francis ${ }^{1} \quad$ M. E. Alkuwari ${ }^{1} \quad$ R. Mohiaddin ${ }^{2} \quad$ C. S. Carr ${ }^{3}$ \\ ${ }^{1}$ Department of Radiology, Heart Hospital, Doha, Qatar \\ 2 Department of Cardiovascular Imaging, Royal Brompton and \\ Address for correspondence C. S. Carr, FRCS(CTh), Heart Hospital, \\ Harefield Hospital, England, United Kingdom \\ ${ }^{3}$ Department of Cardiothoracic Surgery, Heart Hospital, Doha, Qatar \\ Doha, P.O. Box 3050, Qatar (e-mail: ccarr@hamad.qa). \\ Indian J Radiol Imaging 2021;31:751-753.
}
Abstract
Keywords
- hypertrophic cardiomyopathy
- cardiac fibroma
- fibroma

\begin{abstract}
Hypertrophic cardiomyopathy is a relatively common inherited cardiac disorder, with echocardiography still being the initial imaging method for its diagnosis. Cardiac fibroma is a primary neoplasm that most commonly presents in childhood. We present a patient who was investigated for typical cardiac symptoms who went on to have cardiac magnetic resonance imaging and was found to have both of these conditions.
\end{abstract}

\section{Introduction}

Hypertrophic cardiomyopathy (HCM) is a relatively common inherited cardiac disorder with a prevalence of around 1 in 500 in the general population, with echocardiography still being the initial imaging method for its diagnosis. ${ }^{1}$ Cardiac fibroma is a primary neoplasm that most commonly presents in childhood, ${ }^{2}$ with one-third being asymptomatic, but they can also present with symptoms due to obstruction of blood flow, interference with valve function, and arrhythmia (due to pressure on the conduction system). We present a patient who was investigated for typical cardiac symptoms who was found to have both of these conditions.

\section{Case History}

A 37-year-old man presented with a history of intermittent chest heaviness for the preceding 3 years; it had been worsening for 2 months. He also complained of palpitations and light headedness. There was no family history of cardiac disease or sudden death. Examination was normal.

On electrocardiogram, there was sinus bradycardia, T wave inversion in II, III, and aVF, left ventricular hypertrophy, and diffuse peaked T waves. Chest X-ray was unremarkable. The patient proceeded to echocardiography which revealed asymmetrical septal hypertrophy (maximum thickness $2.2 \mathrm{~cm}$ ) with normal wall motion and ejection fraction (57\%). There was no left ventricular outflow tract obstruction or systolic anterior motion of the mitral valve and a there was normal right ventricular function. Computed tomography of the coronary arteries revealed no significant coronary artery disease. A treadmill test and Holter were normal.

Cardiac magnetic resonance imaging (MRI) was performed for suspected HCM. This was performed on a Philips Ingenia 1.5 Tesla Scanner, Eindhoven, and showed classic phenotypic features of HCM with asymmetrical basal septal hypertrophy ( $20 \mathrm{~mm}$ mid-inferoseptal wall vs. corresponding lateral wall $5 \mathrm{~mm}$ ) and patchy mid-wall fibrosis in the hypertrophic segments (-Fig. 1). No left ventricular outflow obstruction or septal anterior motion of the mitral valve was noted. In addition, there was a focal well-defined myocardial thickening/mass in the right ventricular free wall inferiorly measuring $32 \times 21 \mathrm{~mm}$ in maximum dimensions with an initial impression of unusual focal right ventricular hypertrophy related to the hypertrophic cardiomyopathic process (-Fig. 2A, B). On further tissue characterization, the lesion appeared isointense in comparison to the surrounding myocardium on the T1-weighted spin-echo and cine images (balanced steady state in free precession) and hypointense on T2-weighted spin-echo and short tau inversion recovery
DOI https://doi.org/ $10.1055 / \mathrm{s}-0041-1735925$. ISSN 0971-3026.

\footnotetext{
(C) 2021. Indian Radiological Association. All rights reserved. This is an open access article published by Thieme under the terms of the Creative Commons Attribution-NonDerivative-NonCommercial-License, permitting copying and reproduction so long as the original work is given appropriate credit. Contents may not be used for commercial purposes, or adapted, remixed, transformed or built upon. (https://creativecommons.org/ licenses/by-nc-nd/4.0/) Thieme Medical and Scientific Publishers Pvt. Ltd., A-12, 2nd Floor, Sector 2, Noida-201301 UP, India
} 


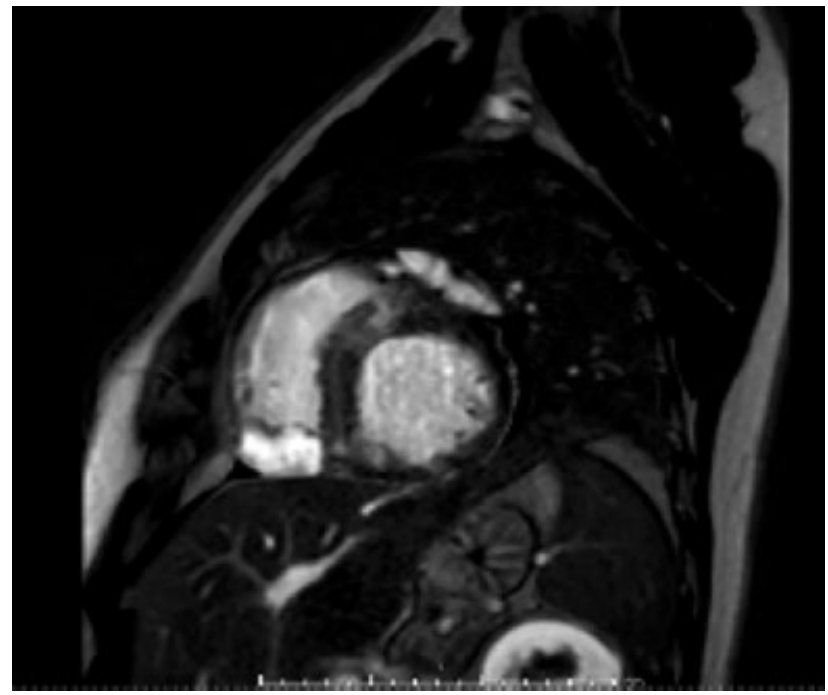

Fig. 1 Cardiac magnetic resonance imaging showed classic phenotypic features of hypertrophic cardiomyopathy with asymmetrical basal septal hypertrophy and patchy mid-wall fibrosis in the hypertrophic segments.

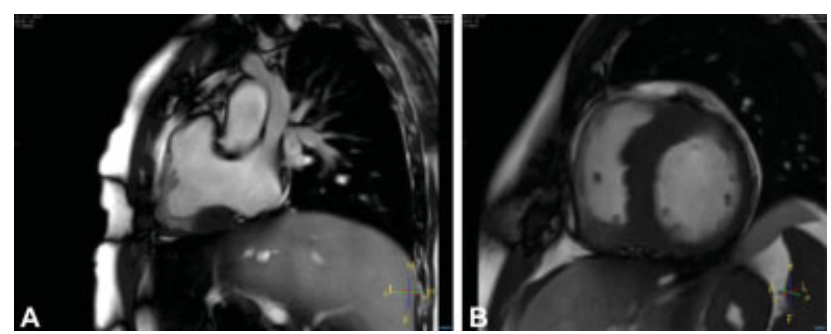

Fig. 2 (A, B) Cardiac magnetic resonance imaging showing a focal well-defined myocardial thickening/mass in the right ventricular free wall inferiorly.

T2 images. Using an inversion-recovery segmented gradient echo sequence, the early phase (less than 3 minutes) after injection of gadolinium contrast, the mass appeared markedly hypointense (-Fig. 3). On late enhancement imaging,

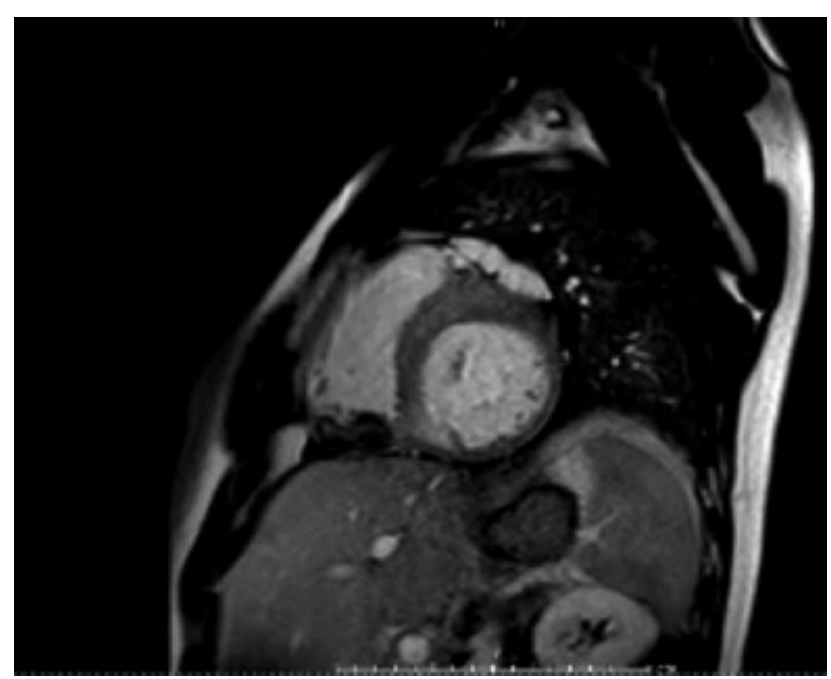

Fig. 3 Inversion-recovery segmented gradient echo sequence, in the early phase, the mass appeared markedly hypointense. performed 10 minutes after contrast injection, the lesion appeared homogeneously hyperintense (-Fig. 1). These findings are typical for cardiac fibroma. A thin layer of myocardium was seen to surround the fibroma.

\section{Discussion}

Sarcomeric HCM is a relatively common inherited cardiac disorder with a prevalence of around 1 in 500 in the general population and is caused by more than 1,400 mutations in 11 or more genes. ${ }^{1}$ The pathologic hallmark is a triad of myocyte hypertrophy, disarray, and myocardial fibrosis. ${ }^{3}$ The disease is characterized by marked heterogeneity with respect to clinical manifestations, natural history, and prognosis, and unfortunately, it is associated with an increased risk of sudden cardiac death ( $\mathrm{SCD}$ ) and progression to heart failure. These three characteristics are however major determinants of SCD. ${ }^{3}$

Two-dimensional echocardiography is still the first-line imaging method for the diagnosis of HCM. However, the quality of images obtained by echocardiography is limited by the available acoustic windows and the orientation of the heart in the mediastinum to ensure adequate cross-sectional images and to avoid oblique views. Furthermore, certain areas of the myocardium are not visualized well with echocardiography, especially the anterolateral free wall in the parasternal short axis view due to poor lateral resolution and substantial image distortion, and detection of subtle areas of abnormal hypertrophy can be missed. ${ }^{4}$ Given that cardiac MRI can be acquired in any given plane, oblique views are avoided, and hypertrophied areas can be visualized and measured with confidence. In a study comparing the incremental benefit of cardiac MRI over echocardiography in the evaluation of patients with HCM, cardiac MRI has a unique ability to determine myocardial tissue characteristics in vivo using the late gadolinium enhancement technique. The presence of fibrosis is an important marker of risk and those patients with greater number of risk factors for SCD typically have more fibrosis, ${ }^{5}$ which is consistent with postmortem data.

Cardiac fibroma is a congenital neoplasm that most commonly presents in childhood. ${ }^{2,6}$ One-third is asymptomatic and detected incidentally, but they can also manifest with symptoms related to obstruction of blood flow, interference with valve function and arrhythmia. Fibromas usually arise from the ventricular septum or the left ventricular free wall, are typically solitary, circumscribed, and often centrally calcified. Histologically, they show a homogenous proliferation of fibroblasts with abundant collagen, which increases with age. ${ }^{2,6,7}$

Echocardiography can confuse cardiac fibroma with $\mathrm{HCM}^{8}$ and it has previously been reported that MRI can distinguish the two conditions. ${ }^{9}$ The hypointense cardiac MRI appearance in the early period following contrast injection reflects the reduced vascularity in comparison to the adjacent myocardium and the hyperintense late enhancement reflects the increased extracellular volume. The tissue characterization provided by cardiac MRI demonstrates the classic cardiac MRI appearances of fibroma and provides accurate diagnosis and potentially avoids the need for more invasive investigations. While the diagnosis of cardiac fibroma using cardiac MRI 
has been reported previously, this case is unusual for involving the right ventricle and in the setting of $\mathrm{HCM}$, its resemblance to right ventricular hypertrophy secondary to HCM.

\section{Disclosures}

No source of funding. Not previously published elsewhere.

\section{Conflict of Interest}

None declared.

\section{Acknowledgment}

The authors would like to acknowledge Mr. Anil Hari, BMRT, PGDHHM, Radiology Technologist, Heart Hospital, Qatar.

\section{References}

1 Maron BJ, Gardin JM, Flack JM, Gidding SS, Kurosaki TT, Bild DE. Prevalence of hypertrophic cardiomyopathy in a general population of young adults. Echocardiographic analysis of 4111 subjects in the CARDIA study. Coronary artery risk development in (young) adults. Circulation 1995;92(04):785-789
2 Basso C, Rizzoi S, Valente M, Thiene G. Prevalence and pathology of primary cardiac tumours. Cardiovasc Med 2012;15(01):18-29

3 Hughes SE. The pathology of hypertrophic cardiomyopathy. Histopathology 2004;44(05):412-427

4 Moon JCC, Fisher NG, McKenna WJ, Pennell DJ. Detection of apical hypertrophic cardiomyopathy by cardiovascular magnetic resonance in patients with non-diagnostic echocardiography. Heart 2004;90(06):645-649

5 O'Hanlon R, Grasso A, Roughton M, et al. Prognostic significance of myocardial fibrosis in hypertrophic cardiomyopathy. J Am Coll Cardiol 2010;56(11):867-874

6 Flores C, Lundberg J, Richardson RR, Prasad D. Utility of cardiac imaging in diagnosis of atypical presentation of cardiac fibroma. BMJ Case Rep 2019;12(09):e230222

7 Chu Z-G, Zhu Z-Y, Liu M-Q, Lv F-J. Cardiac fibromas in the adult. J Card Surg 2014;29(02):159-162

8 Ateńska-Pawłowska JI, Sterliński M, Oręziak A, Sobiech M, Lewandowski M. Left ventricular tumour masquerading as hypertrophic cardiomyopathy: how one can be misled by transthoracic echocardiography. Kardiol Pol 2017;75(03):280

9 Nucifora G, Pasotti E, Pedrazzini G, Moccetti T, Faletra FF, Gallino A. Cardiac fibroma mimicking hypertrophic cardiomyopathy: role of magnetic resonance imaging in the differential diagnosis. Int J Cardiol 2012;154(01):e11-e13 